\title{
A new platform based on IEEE802.15.4 wireless inertial sensors for motion caption and assessment
}

\author{
F. Brunetti, J.C. Moreno, A.F. Ruiz, E. Rocon and J.L. Pons
}

\begin{abstract}
Systems for motion ca;ption and assessment in biomechanics are mostly based on photogrammetry. These systems are restricted to the movement analysis lab and moreover, they are very expensive. New advances in MEMs (Microelectromechanical) and wireless technologies enable inertial sensing as an alternatives for motion caption. This paper presents a wireless inertial sensor including 3 linear accelerometers, 3 gyroscopes and 3 magnetometers. The IMU (inertial measurement unit) includes a IEEE802.15.4 compliant transceiver. The platform expands the frontiers of movement analysis for motion caption in real scenarios like sports and wearable robotics since it does not need structurated labs. Besides the advantages, the cost of the platform is much lower comparing actual photogrammetry systems.
\end{abstract}

\section{INTRODUCTION}

Over the last years, technologies for movement assessment have been widely developed for biomechanics and animation. These systems are usually based on photogrammetry which restricts the workspace to a structurated lab. In spite of this drawbacks, photogrammetry and more specifically systems like VICON and Coda motions are the most common solutions used actually. Another technology that can be used in movement assessment is electrogoniometry. This technology is very common in clinical environments because of its size and the simplicity of the measured variable: the joint angle.

The technologies before commented, have some drawbacks that restrict movement caption in real situations. Monitoring and acquiring in real time activities have become very popular during last years especially promoted by the elder care, sports practice assessment and also motivated by the advances in wearable robotics and mobility.

Inertial sensors based on microelectromechanical systems (MEMS) represent a new alternative for the movement assessment in real scenarios. These sensors have a small size and weight, and a low power consumption. These features enable the development of portable sensors that can be placed on the body segment to be measured. Comparing with the other technologies,inertial sensing offers different features that can be exploited in new applications. Table I shows different qualities of the technologies above commented. Photogrammetry systems are very expensive comparing the other systems, but the setup for a measurement session is a simple process, consisting in a calibration procedure and the placement of markers. Electrogoniometers and inertial sensors have the drawback in the need of a external reference since they measure the angles in their own coordinate

\footnotetext{
The authors are with Instituto de Automtica Industrial, Consejo Superior de Investigaciones Cientficas, Crta Campo Real km 0,200, 28500, Arganda del Rey,Madrid-Spain. brunetti@iai.csic.es
}

TABLE I

MOVEMENT ASSESSMENT TECHNOLOGIES

\begin{tabular}{|c||c|c|c|}
\hline Quality & Photogrammetry & Electrogoniometry & Inertial \\
\hline \hline Cost & High & Medium & Low \\
\hline Precision & High & High & Medium \\
\hline CPU Cost & High & Low & Medium \\
\hline Assembly & Low & Medium & Low \\
\hline Portability & Low & Medium & High \\
\hline Consumption & High & Low & Low \\
\hline Others & Markers occlusion & Ext. Ref. & Ext. Ref. \\
\hline
\end{tabular}

systems while photogrammetry offers the angular positions in an absolute coordinate system.

In spite of this, inertial sensing has become very popular due to its low cost and portability, enabling new movement assessment applications.

\section{INERTIAL SENSORS}

An inertial sensor is usually composed by a set of linear accelerometers [1]. However, there are more complex versions that include besides the accelerometers, a set of gyroscopes [2] and magnetometers [3]. In this way, with this complete set of sensors we have 2 external references under certain conditions: gravity force due to the accelerometers and the magnetic pole due to the magnetometers. With these references in a first approach, we can obtain the angular position integrating the angular velocity provided by the gyroscopes. In real applications obtaining the angular position is not as simple as commented before due to the noise in signals.

\section{A. Accelerometers}

An accelerometer measures the acceleration that the sensor is subject to. The basic setup of a MEMS accelerometer consists in a mass attached to a fixed spring spring [1]. The mass can move in only one direction which is the measured one, that will be consider as the axis of the sensor. The displacement of the mass is a measure of the difference between the acceleration $\vec{g}$ due to the projected gravity force vector in the axis of the sensor $\vec{n}$. The acquired signal relates the magnitude of the resulting vector $\vec{S}$ and can be represented according to:

$$
\vec{S}_{A, n}=k_{A, n}(\vec{a}-\vec{g}) \cdot \vec{n}+o_{A, n},
$$

where $k_{A, n}$ represents a scale factor and $o_{A, n}$ the offset. If we assembly 3 orthogonal accelerometers we obtain a 3D accelerometer. New 3D accelerometers has been released to 
the market months ago (Analog Devices ST Microelectronics). Therefore, when we deal with a 3D accelerometer, in an ideal case 1 can be simplified, after calibration and gain adjustment, as follows:

$$
\vec{S}_{n e t}=\vec{S}_{a}-\vec{S}_{g} .
$$

We use $S$ to emphasize that the lecture is in the sensor coordinate system. The 3D MEMS accelerometer works as an inclinometer in static situations. The inclination can be obtained calculating the angle between the sensor axis and the gravity force vector.

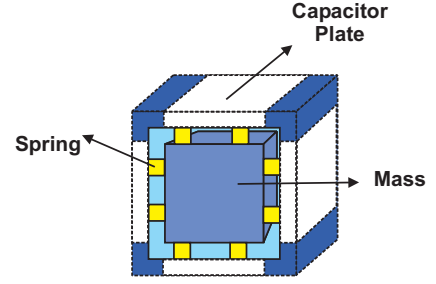

3D Accelerometer

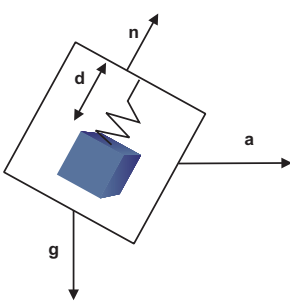

Linear Accelerometer
Fig. 1. Basic scheme of an accelerometers, the first one is the $3 \mathrm{D}$ accelerometer proposed by Lotters

Lotters [4] proposed another type of 3D accelerometer that is shown on the left of Fig. In this configuration, the author uses the variation of the capacitance due to the displacement of the cube surfaces, as a measure of the acceleration.

\section{B. Gyroscopes}

Gyroscopes measure angular velocity. There are three basic types of them: spinning rotor gyroscope, ring laser gyroscopes and vibrating mass gyroscopes. The last one present many advantage for portable applications due to its size, weight, power consumption and cost. An internal mass in constantly vibrating inside the sensor. It takes advantage of the Coriolis effect to measure the angular velocity.

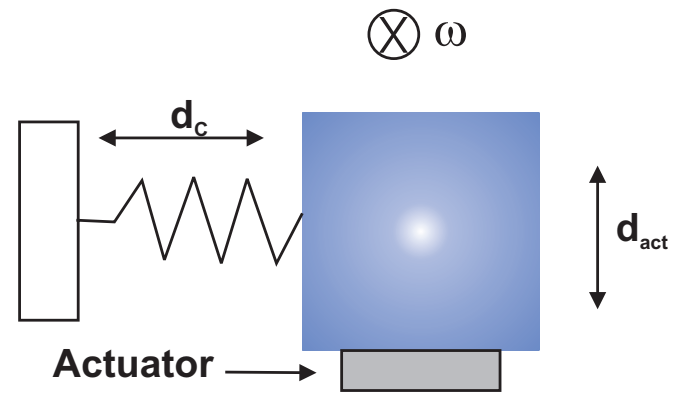

Fig. 2. Scheme of a vibrating mass gyroscope.

Fig. 2 shows the scheme of the sensor. The mass is located over an piezoelectric actuator that cause the vibration in direction $d_{a c t}$ as depicted in fig. 2. The sensor measures the orthogonal mass displacement to $d_{a c t}$. If an angular velocity exists, perpendicular to the sensor plane, the mass will be affected by a force, orthogonal to the plane described by angular velocity vector $\omega$ and the linear velocity of the mass v. The magnitude of this force, the Coriolis Force, is

$$
f_{C}=2 m \cdot v \cdot \omega
$$

where $m$ is the vibrating mass, $v$ the instantaneous linear velocity of the mass, caused by the actuator, and $\omega$ the angular velocity of the sensor. Thus, we have that the measured force is proportional to the angular velocity. As same as accelerometers, a 3D gyroscope can be obtained mounting 3 gyroscopes orthogonally. Unfortunately, until these moments there is not a $3 \mathrm{D}$ gyroscope in a single chip.

\section{Magnetometers}

One of the main problems of $3 \mathrm{D}$ inertial sensing is that it needs external references. Using accelerometers we can use the gravity force as a reference in vertical plane, at least in static situations, but we still do not have a reference for the horizontal plane. This needed reference can be obtained using magnetometers.

Again we can find a solution in MEMS, as show in the scheme of fig. 3. Depending on the orientation of the magnetic field, a torque is generated and can be calculated in order to obtain the angle of incidence of the sensor axis. The f-bOX uses another type of magnetometers, consisting in a magnetoresistive bridge device, the HMC1052, manufactured by Honeywell.

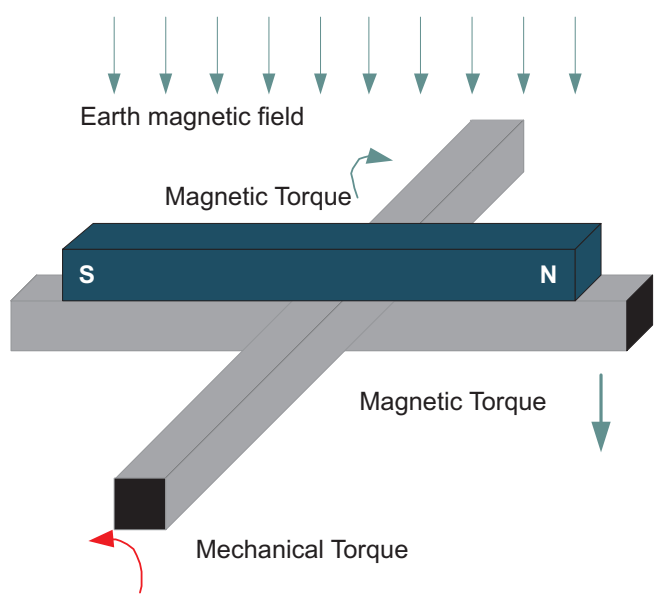

Fig. 3. Operation principle of a MEMS magnetometer.

\section{IEE802.15.4 RADIO}

Inertial sensing can result very complex to manage due to the large quantity of signal that must be handle. This can can result in a wire mess and even more for example if we have to measure many body segments in a biomechanical application. Fortunately, new wireless technologies are available to enable inertial sensing as a solution in movement analysis.

IEEE802.15 task group have been developing several protocols for Wireless Personal Area Networks (WPAN). IEEE802.15.4 is protocol a complementary to the well 
known, Bluetooth (IEEE802.15.1). We describe as complementary since IEEE802.15.4 has a larger range and lower power consumption but as trade-off it has also a lower bitrate.

The f-bOX includes the Chipcon CC2420 radio [5]. It is IEEE802.15.4 compliant transceiver, with a transmission capacity of 250kbps. It can handle short and long IEEE802.15.4 addresses, making it ideal for sensors networks.

\section{F-BOX, WIRELESS INERTIAL SENSOR}

After reviewing the main components of the wireless inertial sensor, we can describe the components used in our design.

The f-bOX (Fun Box) includes: 3 orthogonally mounted gyroscopes (ADXRS300, Analog Devices), a 3D accelerometer (LIS3L02AS4, STMicroelectronics), 2 magnetometers (HMC1052), a microcontroller [6] (ATMEGA128, Atmel) and an ADC (MAX1083, Maxim) with 10 bits of resolution. The f-bOX acquires 10 analog signals, 3 of the accelerometers, 3 of the gyroscopes, 3 for magnetometers and 1 for temperature to compensate possible drifts. The microcontroller also controls the set/reset of the HMC1052 and 3 LEDS as a user feedback. It has implemented a RTC (Real Time Clock) and it is powered with Lithium-ion battery, an Ipod Shuffle(C)one with a charge of $250 \mathrm{mAh}$, enough for a measurement session or a complete game in sports applications.

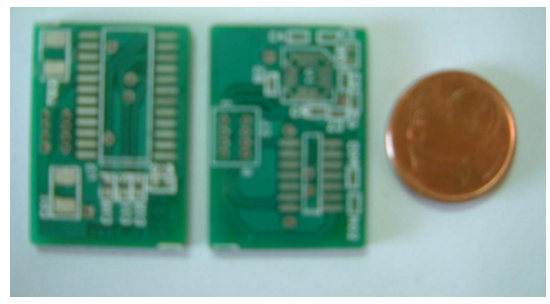

(a)

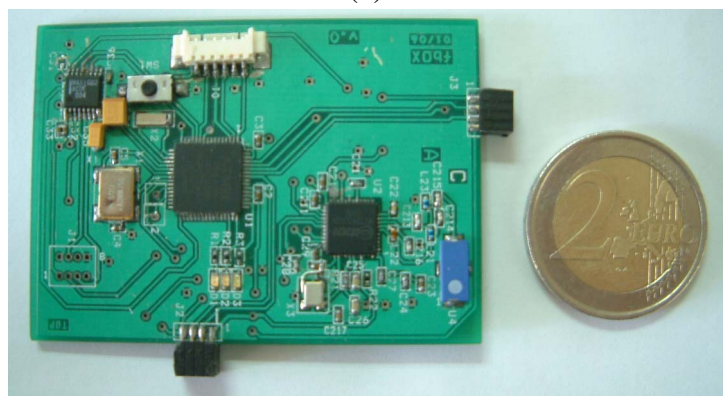

(b)

Fig. 4. f-bOX first prototype. (a) shows one of the sensor board for support one gyroscope and a $3 \mathrm{D}$ accelerometer and (b) the microcontroller and RF section.

The prototypes were manufactured in two layers PCB. Fig. 4 shows the first prototype on on plane sensor board in (a) and the microcontroller and RF section in (b). Future version will be developed using 4 and 6 layers PCB, respectively. Microstrain offers a IEEE802.15.4 inertial sensor but it does not include magnetometers in its configuration [7].

\section{APPLICATION SCENARIOS}

The interest of the develop of this sensor network is twofold. First, we look for an alternative for current movement assessment technologies. The new tool has to be portable in order to make the movement analysis in real scenarios. The second goal is the development of a complete set of tools, to use in wearable robotics. The f-bOX will be just a part of this system. At this moment we have proposed two well defined scenarios that we describe next.

\section{A. Sports practice monitoring}

Collective sports is the first scenario of interest. Wheelchair basketball is the chosen one. Movement analysis during sport practice is almost impossible using current technologies based on photogrammetry. In this application, the platform architecture and the network will handle some hierarchy to improve the throughput of the platform.

In this application we want to assess the movement of the player's upper limb. Using four sensors we can obtain the angular position of each joint: the wrist, the elbow and the shoulder, as depicted in fig. 5. The base station can access any subnet which is composed by the set of inertial sensors of one player. There is a master in every subnet which calculates the angular position of every joint of the player's upper limb. In this way the maximum traffic between nodes is five times the information of each inertial sensor.

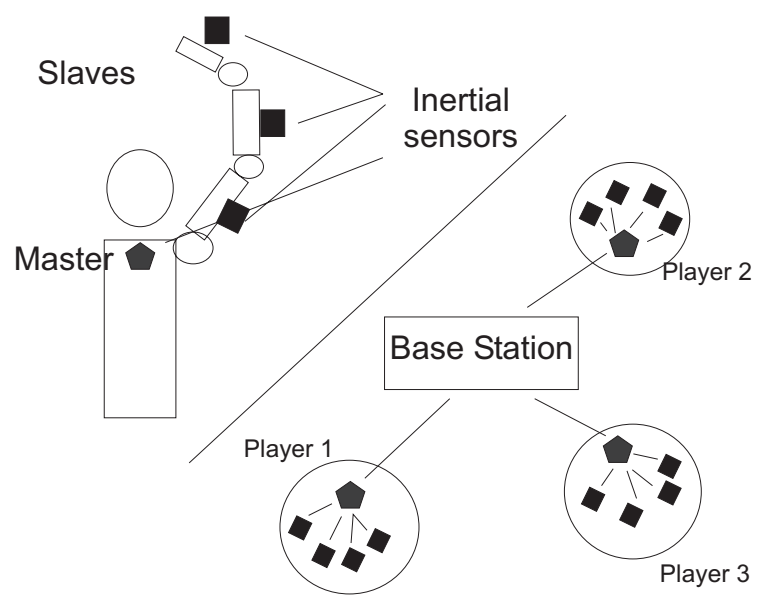

Fig. 5. Movement analysis in wheelchair basketball. On the right of the we can see the network topology to optimize the data throughput.

\section{B. Wearable Robotics}

The second scenario of interest is in the field of wearable robotics. Previous work done by the research group used inertial sensors for movement assessment both in upper and lower exoskeletons. In the framework of the european DRIFTS project an upper limb exoskeleton was developed to cancel pathologic tremor [8]. The wearable robot uses gyroscope to assess tremor. On the other hand, european GAIT project uses gyroscopes and accelerometers to detect gait events [9] in a lower limb orthosis for functional compensation. 
The new approach, in the framework of NEUROLAB project, consists in an upper limb exoskeleton and a complete set of tools, including EEG and EMG [10] tools to assess neuromotor control strategies. Inertial sensing is the only reliable technology for movement assessment in wearable robotics due to size and environment restrictions. Moreover, control requirements demand specific QoS features.

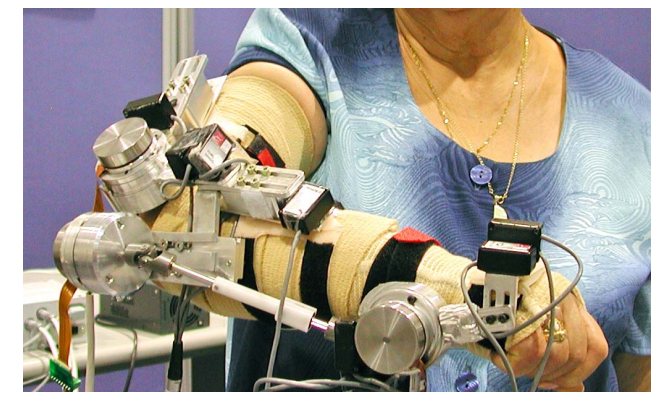

(a)

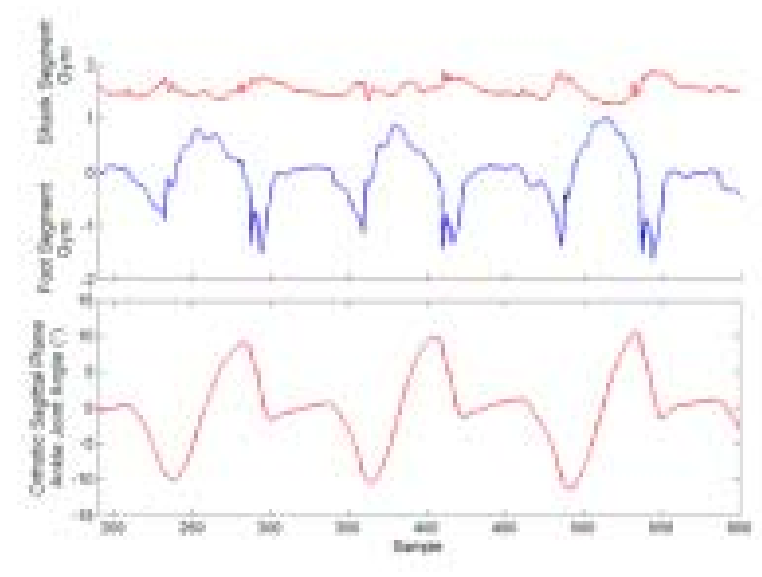

(b)

Fig. 6. Examples of inertial sensors in wearable robotics. (a) shows gyroscopes in DRIFTS exoskeleton and (b), signals describing gait patterns using a lower limb orthosis in the framework of the GAIT project.

\section{CONCLUSIONS}

New technologies, in sensors and wireless networks, enable inertial sensing as an alternative for movement analysis. Current photogrammetry systems are expensive and restricted lab while inertial sensors are low cost and portable.

IEEE802.15.4 is a low power and low cost wireless communication protocol ideal for portable applications. The f-bOX joins these two promising technologies. It is the backbone of the proposed platform described in this paper. Real applications and scenarios now can be boarded using this new platform. The first scenario, collective sport analysis, imposes a hierarchical architecture in order to improve the performance of the sensor network, while the second scenario, wearable robotics, shows the need of smart protocols to cooperate with multisensorial nodes.

\section{FUTURE WORK}

The project is in a first stage. The nodes will be optimized during nest months. PCBs will de designed again using multilayer technology. Anyway, main efforts will be focused on the development of network protocols such service discovery, synchronization and routing, in order to optimize the platform. In the framework of the NEUROLAB project, many different modules are being developed. EEG module EMG and Data Loggers modules are some of the devices part of the whole set of neuro-tools.

Node location is another challenge that will be boarded. Location in very important in collective sports. Chipcon offers a family of IEEE802.15.4sigle chip solution with support for location applications. These chips are an alternative that will be studied.

\section{REFERENCES}

[1] H. Luinge, "Inertial sensing of human movement," Ph.D. dissertation, University of Twente, 2002.

[2] J. Moreno, E. Rocon, A. Ruiz, F. Brunetti, and J. Pons, "Design of an inertial measurement unit for gait kinematics sensing in a lower leg orthosis," in Eurosensor XIX, 2005.

[3] Xsens. [Online]. Available: http://www.xsens.com/

[4] J. L. et al., "Design, fabrication and characterization of a highly symmetrical capacitive triaxial accelerometers," Sensors and Actuators, vol. 66, pp. 205-212, 1998.

[5] Chipcon. [Online]. Available: http://www.chipcon.com/

[6] Atemga128, 8 bit AVR Microcontroller with $128 \mathrm{~KB}$ In-system Programmable Flash, Preliminary ed., Atmel, 2003.

[7] Microstrain. [Online]. Available: http://www.microstrain.com/

[8] J. B.-L. E. Rocon, J. Snchez-Lacuesta, and J. Pons, "Pathological tremor management:modelling, compensatory technology and evaluation," Journal of Technology and Disability, vol. Aceptado, 2005.

[9] J. Moreno, F. Brunetti, and J. Pons, "An autonomous control and monitoring system for a lower limb orthosis : The gait project case," in Proceedings of the 26th International Conference of the IEEE/Engineering in Medicine and Biology Society, 2004.

[10] F. Brunetti, E. Rocon, J. Pons, and M. Manto, "The tremor coherence analyzer(tca): A portable tool to assess instantaneous intermuscle coupling in tremor," in 26th International Conference of the IEEE/Engineering in Medicine and Biology Society, 2004. 